\title{
HIGHER-ORDER SLIDING MODE DYNAMICS DESIGN FOR A CLASS OF SINGLE-INPUT LINEAR SYSTEMS
}

\author{
$U D C(681.5 .01:(531.39+519.87))$
}

\begin{abstract}
Boban Veselić
University of Niš, Faculty of Electronic Engineering, Department of Control Systems, Republic of Serbia
\end{abstract}

\begin{abstract}
The paper considers a higher-order sliding mode dynamics design in a class of single-input linear systems having the invertible system matrix. The proposed sliding manifold selection method simultaneously provides a necessary relative degree of the sliding variable for a specific sliding mode order and the desired system dynamics after establishing that sliding mode. It is shown that the found unique solution satisfies these requirements. The theoretically obtained result is validated through a numerical example and illustrated by digital simulations.
\end{abstract}

Key words: higher-order sliding mode, sliding manifold design, sliding mode dynamics, pole placement.

\section{INTRODUCTION}

Variable structure control systems (VSCS) with sliding modes (SM) [1,2] are one of the more important robust control techniques due to their theoretical invariance to system perturbations that satisfies so called matching conditions [3]. This property in practical implementations is reduced to strong robustness to parameter and external disturbances. The main obstacle in a wider application of this control strategy is the occurrence of chattering due to the existence of system imperfections and unmodeled dynamics. This unwanted effect represents the occurrence of high frequency oscillations, inadmissible in some systems.

Although in most control tasks of plants classified as linear dynamical systems, linear controllers work well, the SM control (SMC) still shows a superior behavior through the overshoot elimination, fast response and excellent robustness to parametric and external disturbances. In addition, the design of conventional SMC of linear systems is systematized through well-defined procedures. Thus, the application of SMC in linear systems is easily implemented and that is why SMC is most frequently applied in these systems. Furthermore, systematic procedures for the sliding manifold (usually hyperplane) design have been also

Received August 05, 2020

Corresponding author: Boban Veselić

University of Niš, Faculty of Electronic Engineering, Aleksandra Medvedeva 14, 18000 Niš, Republic of Serbia

E-mail: boban.veselic@elfak.ni.ac.rs 
developed for linear systems. For these reasons the forthcoming considerations are limited to linear systems only.

The concept of a higher order sliding mode (HOSM) [4] has emerged in attempts to reduce chattering. HOSM control systems were firstly analyzed in a single input system, and then in multiple input systems as well as discrete-time systems [5-8]. The most important results have been established in case of the second order SM [9,10].

An initial step in the SM control system design is selection of a sliding manifold, defined by a sliding variable. This defines the desired system dynamics in SM along the sliding manifold. In conventional SM (first order SM), the relative degree of the sliding variable with respect to control must be one. Several sliding manifold design methods were proposed that provide desired dynamics in the first order SM. One approach is the system transformation into so called regular form [2] in which the reduced SM dynamics is easily noticeable. In case of single input linear systems, it is possible to design the sliding manifold without the system transformation in a way that is based on the application of the Ackermann's formula [11]. Also, a simple comprehensive sliding manifold design method for multiple input linear systems was developed not needing the system transformation [12,13].

In case of HOSM, the sliding variable relative degree must be equal to the SM order. Hence, the design of a sliding manifold needs to accomplish a twofold task: to provide the desired reduced order dynamics in HOSM as well as to fulfill the precondition of the required sliding variable relative degree. There is a small number of papers that deal with this problem, and so far only single input linear systems are considered. Generalization of the Ackermann-Utkin formula [11] for a sliding manifold design in single-input systems in case of an arbitrary order SM was done in [14,15].

The main motivation behind this paper was to investigate if the comprehensive approach to sliding manifold design systems [13], developed for conventional (or first-order) SMs, could be utilized in HOSM. The paper proposes a way of HOSM dynamics design for a class of single input linear systems of $n$-th order. This method relies on the conventional state feedback control design approach. Besides the requirement to ensure a predefined desired $(n-r)$-th order dynamics in the $r$-th order SM, the sliding variable selection must also satisfy the precondition to have the relative degree $r$. A simple solution for the vector $c$ defining the sliding variable is found in case of systems having a full rank system matrix. It is proved that such designed sliding manifold provides both requirements. The validity of the proposed solution has been tested in a numerical example and illustrated by simulation results.

\section{PRELIMINARIES AND PROBLEM STATEMENT}

Consider a linear time-invariant single-input system described by the following state space model

$$
\dot{x}=A x+b(u+d),
$$

where $x \in \mathbb{R}^{n}$ is the available state vector and $u, d \in \mathbb{R}$ are the control and an unknown bounded disturbance, respectively. $A$ and $b$ are constant matrices of appropriate dimensions and the system is fully controllable, i.e. the controllability matrix $Q_{c}=$ $\left[\begin{array}{llll}b & A b & \cdots & A^{n-1} b\end{array}\right]$ has a full rank $\left(\operatorname{rank} Q_{c}=n\right)$. Obviously, the disturbance satisfies the matching condition [3] since it affects the system through the input channel. Notice that all variables in (1) are functions in time, but the time arguments have been omitted due to 
a shorter notation hereafter. The task is to organize the $r$-th order sliding mode in the system (1) having a desired sliding mode dynamics.

Let the sliding variable $g \in \mathbb{R}$ be defined as

$$
g=c x, c \in \mathbb{R}^{1 \times n} .
$$

The system motion defined by (1) and (2) along the manifold

$$
g=\dot{g}=\ddot{g}=\cdots=g^{(r-1)}=0
$$

denotes the $r$-th order sliding mode, [6]. Control that in the system (1), (2) ensures the condition (3) in finite time must be discontinuous [8], at least in the set (3), under the condition that the sliding variable has a relative degree $r$ with respect to control. This means that the control signal occurs only in the $r$-th time derivative of $g$, i.e. in $g^{(r)} \cdot r$-th time derivative of the sliding variable is obtained as

$$
g^{(r)}=c A^{r} x+\sum_{j=0}^{r-1} c A^{r-1-j} b\left(u^{(j)}+d^{(j)}\right) .
$$

The condition that the sliding variable has required relative degree can be expressed as

$$
\begin{gathered}
c \cdot\left[\begin{array}{cccc}
b & A b & \cdots & A^{r-2} b
\end{array}\right]=0, \\
c A^{r-1} b \neq 0 .
\end{gathered}
$$

The condition (6) is usually fulfilled by the selection $c A^{r-1} b=1$ in order to provide further simplifications. Then, the $r$-th time derivative of the sliding variable (4) becomes

$$
g^{(r)}=c A^{r} x+u+d
$$

In the $r$-th order sliding mode, the sliding mode dynamics is of $(n-r)$-th order [9], which can be found using an equivalent control method. The equivalent control can be obtained from the condition

$$
\left.g^{(r)}\right|_{u=u_{e q}}=0
$$

which gives

$$
u_{e q}=-c A^{r} x-d
$$

The replacement of the equivalent control in (1) gives the system description in the $r$-th order sliding mode

$$
\dot{x}=A x+\left.b u\right|_{u=u_{e q}}=\left(I-b c A^{r-1}\right) A x=P A x=A_{e q} x .
$$

Sliding mode dynamics (10) in the ideal $r$-th order sliding mode is not affected by the disturbance $d$, so the system is invariant to the matched disturbances. However, the equivalent control (9) is not feasible since it requires the knowledge of disturbances. Also, it is easy to prove that the matrix $P$ is idempotent, i.e. it holds $P^{2}=P$. This means that $P$ is a projector. Due to projector matrices properties [16], it follows that $\operatorname{rank}(P)=n-1$. Under assumption $\operatorname{rank}(A)=n$ it holds $\operatorname{rank}\left(A_{\text {eq }}\right)=\operatorname{rank}(P A)=n-1$, which means that $\operatorname{det}\left(A_{e q}\right)=0$ and $A_{e q}$ is a singular matrix. Obviously, eigenvalues of the matrix $A_{e q}$ define sliding mode dynamics, which needs to be stable. 
Therefore, the vector $c$ should be determined that provides the desired $r$-th order sliding mode dynamics (10) as well as the required relative degree $r$ of the sliding variable (2). A simple way of selection of $c$ is suggested in the next section.

\section{THE MAIN RESULT}

Since the equivalent control (9) is linear and state dependent, it can be considered as a traditional state feedback control. Namely, the model (10) that describes sliding mode dynamics can be rewritten as

$$
\dot{x}=A_{e q} x=\left(A-b c A^{r}\right) x=(A-b k) x,
$$

where $k \in \mathbb{R}^{1 \times n}$ is the gain vector of the state feedback control $u=-k x$. Sliding mode dynamics in case of the $r$-th order sliding mode is characterized by $r$ zero and $n-r$ nonzero eigenvalues. Nonzero eigenvalues $\lambda_{1}, \lambda_{2}, \ldots, \lambda_{n-r}$ should be chosen to be stable and to define the desired sliding mode dynamics, which can be described by the following characteristic polynomial

$$
\begin{gathered}
\phi(s)=s^{r}\left(s-\lambda_{1}\right)\left(s-\lambda_{2}\right) \cdots\left(s-\lambda_{n-r}\right)=s^{r} \phi_{r}(s), \\
\phi_{r}(s)=\left(s-\lambda_{1}\right) \cdots\left(s-\lambda_{n-r}\right)=\sum_{i=0}^{n-r} \beta_{i} s^{n-r-i}, \beta_{0}=1 .
\end{gathered}
$$

It is well known that a unique state feedback gain vector $k$ exists in the controllable system (11) that will provide the desired dynamics (12). A possible way to find $k$ is to je apply the Ackermann's formula

$$
k=\left[\begin{array}{ll}
0_{1 \times(n-1)} & 1
\end{array}\right] Q_{c}^{-1} \phi(A)=\left[0_{1 \times(n-1)} \quad 1\right] Q_{c}^{-1} \phi_{r}(A) A^{r} .
$$

Such obtained $k$ can be used for finding an unknown vector $c$. According to (11), a relationship between $c$ and $k$ can be noticed, which is given by the relation $c A^{r}=k$. The vector $c$ that satisfies this equation will provide the desired dynamics (12) in the $r$-th order sliding mode. However, beside this requirement, $c$ should provide the required relative degree of the sliding variable with respect to control as well. Hence, $c$ must satisfy the following equations

$$
\begin{gathered}
c A^{r}=k, \\
c \cdot\left[\begin{array}{llll}
b & A b & \cdots & A^{r-2} b
\end{array}\right]=0 .
\end{gathered}
$$

The system of equations that is formed out of (14) and (15) consists of $n+r-2$ scalar equations with $n$ unknown components of the vector $c$. The next theorem gives the unique solution of the obtained system of equations.

Theorem 1: If the matrix $A$ of the controllable system (1) has a full $\operatorname{rank}$, i.e. $\operatorname{rank}(A)=$ $n$, then

$$
c=k A^{-r}
$$

is the unique solution of the equations (14) and (15).

Proof: If $\operatorname{rank}(A)=n$ then $\operatorname{rank}\left(A^{r}\right)=n$ and inverse matrix $A^{-r}$ exists. Obviously, solution (16) fulfils equation (14). However, it is necessary to prove that solution (16) simultaneously satisfies equation (15). Replacement of (16) into $c\left[\begin{array}{llll}b & A b & \cdots & A^{r-2} b\end{array}\right]$ by virtue of (13) yields 


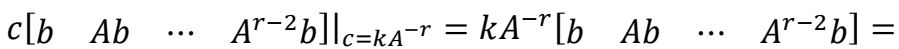

$\left[0_{1 \times(n-1)} 1\right] Q_{c}^{-1} \phi_{r}(A)\left[\begin{array}{llll}b & A b & \cdots & A^{r-2} b\end{array}\right]$.

For $\phi_{r}(A)=\sum_{i=0}^{n-r} \beta_{i} A^{n-r-i}, \beta_{0}=1$, it is obtained

$\left.c\left[\begin{array}{llll}b & A b & \cdots & A^{r-2} b\end{array}\right]\right|_{c=k A^{-r}}=$

$\left[\begin{array}{ll}0_{1 \times(n-1)} & 1\end{array}\right] Q_{c}^{-1} \sum_{i=0}^{n-r} \beta_{i} A^{n-r-i}\left[\begin{array}{llll}b & A b & \cdots & A^{r-2} b\end{array}\right]=\left[\begin{array}{lll}0_{1 \times(n-1)} & 1\end{array}\right] Q_{c}^{-1} \sum_{i=0}^{n-r} \beta_{i}$.

$\left[\begin{array}{llll}A^{n-r-i} b & A^{n-r-i+1} b & \cdots & A^{n-i-2} b\end{array}\right]=\left[\begin{array}{ll}0_{1 \times(n-1)} & 1\end{array}\right] \cdot \sum_{i=0}^{n-r} \beta_{i}$.

$\left[Q_{c}^{-1} A^{n-r-i} b \quad Q_{c}^{-1} A^{n-r-i+1} b \quad \cdots \quad Q_{c}^{-1} A^{n-i-2} b\right]$.

The product $A^{j} b, j=0,1, \ldots, n-1$ can be extracted from the controllability matrix $Q_{c}$ in the following manner

$$
A^{j} b=Q_{c} \cdot\left[\begin{array}{c}
0_{j \times 1} \\
1 \\
0_{(n-j-1) \times 1}
\end{array}\right], j=0,1, \ldots, n-1 .
$$

It follows accordingly

$$
Q_{c}^{-1} A^{j} b=\cdot\left[\begin{array}{c}
0_{j \times 1} \\
1 \\
0_{(n-j-1) \times 1}
\end{array}\right], j=0,1, \ldots, n-1 .
$$

Applying (20) onto the matrix elements in (18) it is obtained

$$
\left.c\left[\begin{array}{llll}
b & A b & \cdots & A^{r-2} b
\end{array}\right]\right|_{c=k A^{-r}}=\left[\begin{array}{lllll}
0_{1 \times(n-1)} & 1
\end{array}\right] \cdot\left[\begin{array}{cccc}
\beta_{n-r} & 0 & \cdots & 0 \\
\beta_{n-r-1} & \beta_{n-r} & \cdots & 0 \\
\vdots & \beta_{n-r-1} & \cdots & \vdots \\
\beta_{0} & \vdots & & 0 \\
0 & \beta_{0} & \cdots & \beta_{n-r} \\
0 & 0 & \cdots & \beta_{n-r-1} \\
\vdots & \vdots & & \vdots \\
0 & 0 & \cdots & \beta_{0} \\
0 & 0 & \cdots & 0
\end{array}\right]
$$

Since the last row of the obtained matrix is zero, the right hand side product of (21) is equal to $0_{1 \times(r-1)}$. This shows that solution (16) satisfies equation (15), which completes the proof.

\section{HOSM CONTROLLER}

It was shown in [6] that the control in the system (1), (2) that provides the high order sliding mode (3) in finite time must be discontinuous, at least in the set (3). If the sliding mode dynamics is stable, which is set by the adequate selection of $c$, the system trajectory asymptotically converges along (3) into the origin $(x \rightarrow 0$ for $t \rightarrow \infty)$, in spite of the action of the matched disturbances.

If the control in the system (1), (2) is chosen as in [7]

$$
u=-c A^{r} x-\gamma(\xi), \xi=\left(g, \dot{g}, \ldots, g^{(r-1)}\right),
$$


$r$-th time derivative of $g(7)$ becomes

$$
g^{(r)}=-\gamma(\xi)+d
$$

Levant proposed in [6] a set of quasi-continuous functions $\gamma(\xi)$ that enforces $r$-th order sliding mode in single-input nonlinear systems. Complexity of these functions increases with the increase of the sliding mode order $r$, while chattering decreases. These functions can be applied for the linear case (23).

Functions $\gamma(\xi)$ that guarantee the higher order sliding mode occurrence in finite time can be chosen as nonlinear functions proposed in [5,6]. For example, functions $\gamma(\xi)$ for $r=1,2,3$ are respectively given as

$$
\begin{gathered}
\gamma(\xi)=\alpha \frac{g}{|g|^{2}}, \\
\gamma(\xi)=\alpha \frac{\dot{g}+\beta_{1}|g|^{\frac{1}{2}} \operatorname{sign}(g)}{|\dot{g}|+\beta_{1}|g|^{\frac{1}{2}}}, \\
\gamma(\xi)=\alpha \frac{\ddot{g}+\beta_{2}\left(|\dot{g}|+\beta_{1}|g|^{\frac{2}{3}}\right)^{-\frac{1}{2}}\left(\dot{g}+\beta_{1}|g|^{\frac{2}{3}} \operatorname{sign}(g)\right)}{|\ddot{g}|+\beta_{2}\left(|\dot{g}|+\beta_{1}|g|^{\frac{2}{3}}\right)^{\frac{1}{2}}},
\end{gathered}
$$

where $\alpha, \beta_{1}, \beta_{2}>0$ are chosen sufficiently large.

It is important to notice that realization of $\gamma(\xi)$ requires the knowledge of the successive derivatives of the sliding variable, i.e. $g^{(i)}, i=0,1, \ldots, r-1$. However, in the systems with matched disturbances these derivatives according to (4) and (5) can be obtained as

$$
g^{(i)}=c A^{i} x, i=0,1, \ldots, r-1 .
$$

In this case, the need for real differentiators is unnecessary.

\section{AN ILLUSTRATIVE EXAMPLE}

The proposed higher sliding manifold design method and its validity has been demonstrated on a numerical example and illustrated by digital simulations. Consider a fully controllable fifth order system (1) whose matrices are

$$
\begin{aligned}
& A= {\left[\begin{array}{ccccc}
-1.129 & -2.262 & 2.21 & 0.465 & 1.663 \\
1.829 & -0.743 & -1.344 & -3.802 & -6.199 \\
-1.06 & 1.87 & -1.375 & -2.324 & 0.181 \\
0.399 & 4.190 & 1.258 & -1.062 & 0.908 \\
-2.89 & 5.645 & 1.334 & 0.24 & -1.45
\end{array}\right], } \\
& b^{\mathrm{T}}=\left[\begin{array}{llllll}
0.4136 & 0 & 0.144 & 0 & -0.7601
\end{array}\right] .
\end{aligned}
$$

The system is subjected to a matched external disturbance in a complex form $d(t)=$ $2 \sin (4 \pi t)+2 h(t-3)$. The control task is to bring the system state from an arbitrarily chosen initial state $x(0)=\left[\begin{array}{lllll}1 & 1 & 1 & 1 & 1\end{array}\right]^{\mathrm{T}}$ into the origin regardless of the disturbance by organizing the second order sliding mode. Since in this case $n=5$ and $r=2$, the sliding mode dynamics is of order $n-r=3$. Hence, let a desired system dynamics be defined by the 
spectrum of poles $p=\left[\begin{array}{lllll}0 & 0 & -3 & -3 & -3\end{array}\right]$. According to (13), state feedback gain $k$ is obtained as

$$
k=\left[\begin{array}{lllll}
4.2365 & -12.9816 & -3.4063 & -0.8998 & -2.6053
\end{array}\right]
$$

The corresponding value of the vector $c$ defining the sliding manifold is found using formula (16) as

$$
c=\left[\begin{array}{lllll}
0.052 & 0.2238 & 0.101 & 0.0976 & 0.0474
\end{array}\right]
$$

Calculations confirmed that the obtained $c$ satisfies equations (14), (15) and that the matrix $\left(A-b c A^{r}\right)$ in (11) has the desired eigenvalues spectrum $p$.
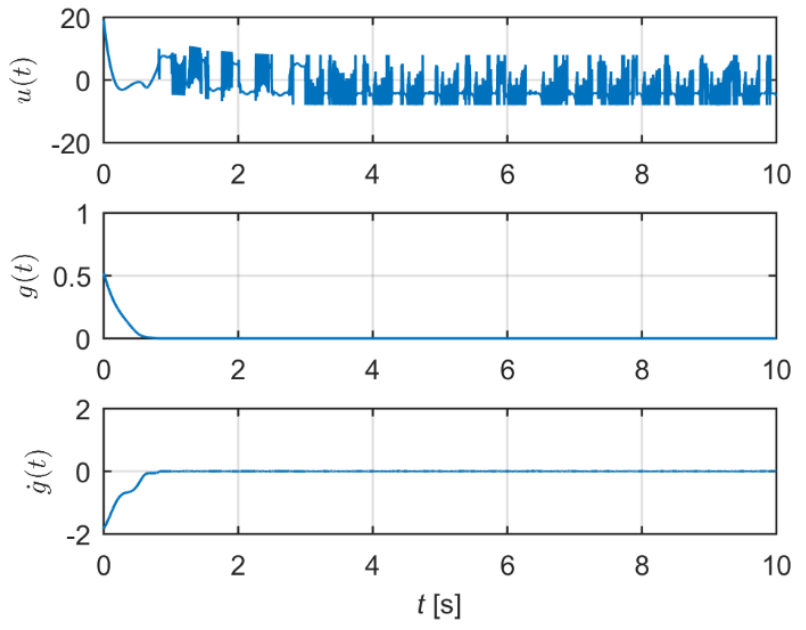

Fig. 1 Control signal, sliding variable and its derivative

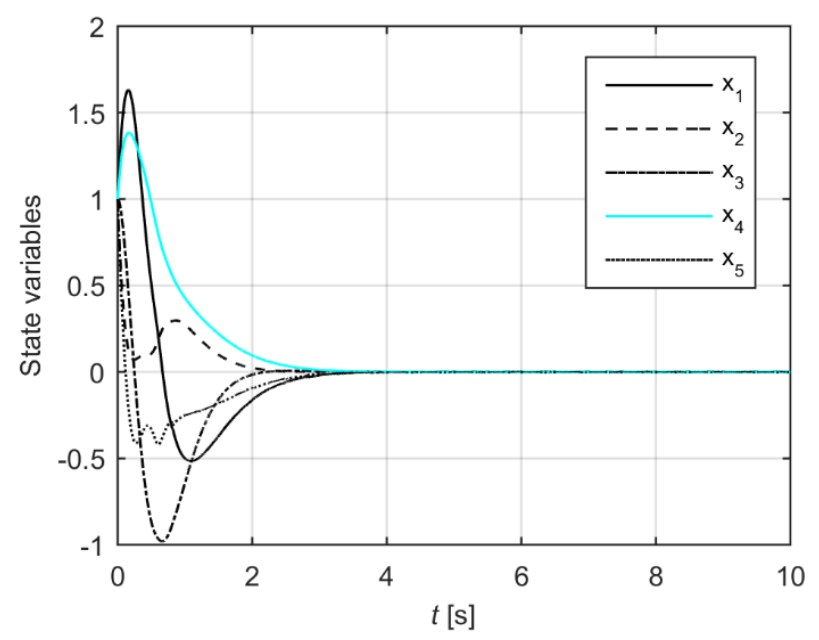

Fig. 2 State variables 
The control that provides the occurrence of the second order sliding mode in the considered system is defined by (22) and (25). The required sliding variable first order derivative $\dot{g}$ is estimated using (27). The controller parameters are chosen as $\alpha=8$ and $\beta_{1}=$ 1. Fig. 1. shows the control signal $u(t)$, sliding variable $g(t)$ and its derivative $\dot{g}(t)$. It is evident that the control ensures $g=\dot{g}=0$, i.e. the second order sliding mode in finite time regardless of the disturbance. Fig. 2 . shows that the sliding variables asymptotically converge into the origin after establishing the sliding mode, with the prescribed dynamics defined by $p$.
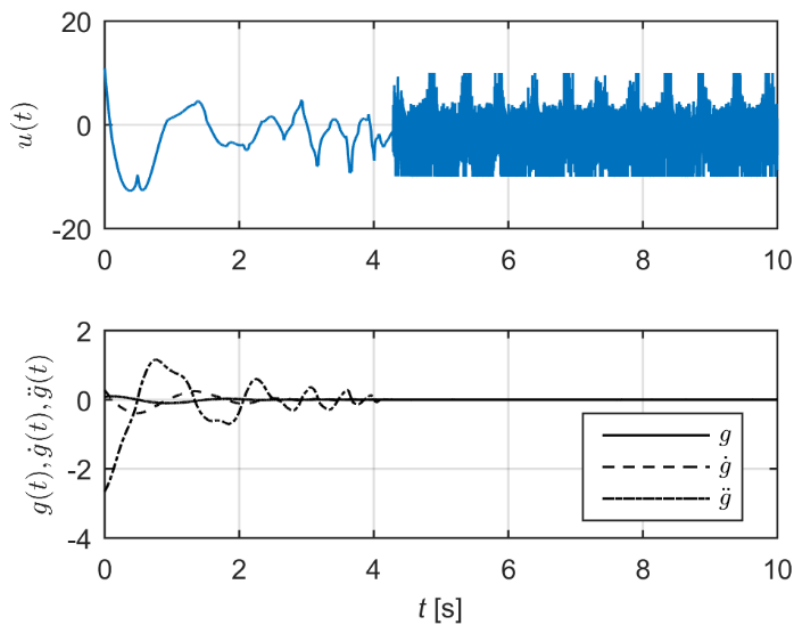

Fig. 3 Control signal, sliding variable and its derivatives

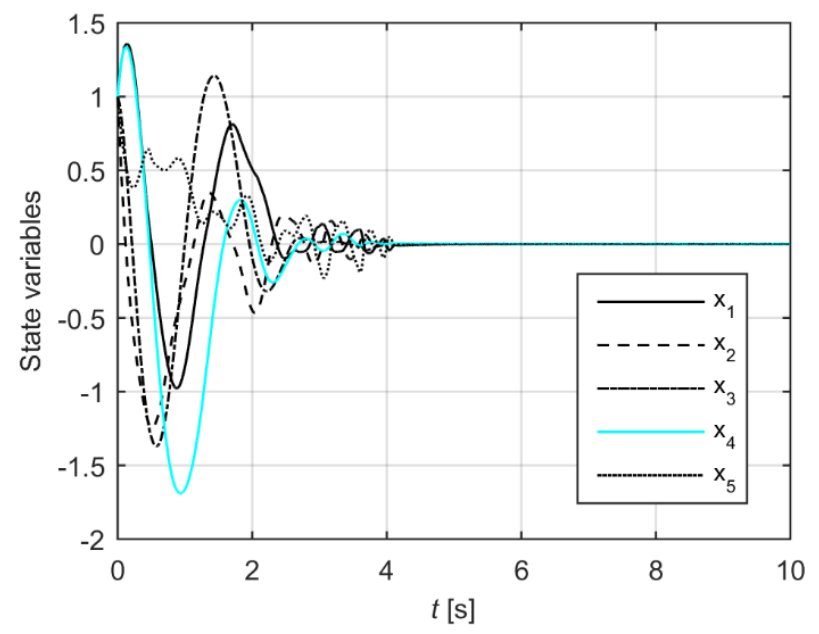

Fig. 4 State variables 
Now, let the desired sliding mode order be $r=3$. Then the sliding mode dynamics is the second order and let the desired dynamics be defined by the spectrum $p=$ $\left[\begin{array}{lllll}0 & 0 & 0 & -3 & -3\end{array}\right]$. The corresponding state feedback gain $k$ is obtained according to (13) as

$$
k=\left[\begin{array}{lllll}
3.7024 & -12.9186 & -1.8852 & 2.0798 & 1.3391
\end{array}\right]
$$

The required sliding manifold vector $c$ is calculated using (16) as

$$
c=\left[\begin{array}{lllll}
0.021 & 0.0076 & -0.0073 & 0.0503 & 0.01
\end{array}\right],
$$

which satisfies (14) and (15) and ensures that the matrix $\left(A-b c A^{r}\right)$ in (11) has the desired eigenvalues spectrum $p$.

The control that provides the occurrence of the third order sliding mode in the considered system is defined by (22) and (26). The required derivatives $\dot{g}$ and $\ddot{g}$ are estimated using (27). The controller parameters are chosen as $\alpha_{1}=10, \beta_{1}=1$ and $\beta_{2}=$ 2 , and the system is subjected to the identical disturbance. Control signal $u(t)$, sliding variable $g(t)$ and its derivatives $\dot{g}(t)$ and $\ddot{g}(t)$ are depicted in Fig. 3. Since the control ensures $g=\dot{g}=\ddot{g}=0$, the third order sliding mode occurs in finite time in spite of the disturbance. Fig. 4 . shows the sliding variables that asymptotically converge into the origin with the prescribed dynamics defined by $p$.

\section{CONCLUSION}

The proposed sliding manifold design method for the higher order sliding mode singleinput control systems relies on the conventional state feedback control design principle. The method, which can be applied in systems having a full rank system matrix, does not require system transformation nor extensive calculations. It is proved that the designed vector $c$ ensures the desired dynamics in the higher order sliding mode and the appropriate sliding variable relative degree. Validity of the analytically obtained solution has been confirmed through numerical example and simulations.

The given approach to the sliding manifold design can be entirely applied in discrete time higher order sliding mode regimes.

Acknowledgement: This work has been supported by the Ministry of Education, Science and Technological Development of the Republic of Serbia.

\section{REFERENCES}

[1] V. I. Utkin, "Variable structure systems with sliding mode," IEEE Transactions on Automatic Control, vol. 22, no. 2, pp. 212-222, 1977. [Online]. Available: http://dx.doi.org/10.1109/TAC.1977.1101446

[2] V. I. Utkin, Sliding modes in control and optimization, Berlin, Germany: Springer-Verlag, 1992. [Online]. Available: http://dx.doi.org/10.1007/978-3-642-84379-2

[3] B. Draženović, "The invariance conditions in variable structure systems," Automatica, vol. 5, no. 3, pp. 287-295, 1969. [Online]. Available: http://dx.doi.org/10.1016/0005-1098(69)90071-5

[4] A. Levant, "Sliding order and sliding accuracy in sliding mode control," Int. J. Contr., vol. 58, no. 6, pp. 1247-1163, 1993. [Online]. Available: http://dx.doi.org/10.1080/00207179308923053

[5] A. Levant, "Homogeneity approach to higher-order sliding mode design" Automatica, vol. 41, pp. 823830, 2005. [Online]. Available: http://dx.doi.org/10.1016/j.automatica.2004.11.029 
[6] A. Levant, "Quasi-continuous high-order sliding-mode controllers," IEEE Trans. on Automatic Control, vol. 50, no. 11, pp. 1812-1816. 2005. [Online]. Available: http://dx.doi.org/10.1109/TAC.2005.858646

[7] V.I. Utkin, "Discussion aspects of higher-order sliding modes," IEEE Trans. on Automatic Control, vol. 61, no. 3, pp. 829-833, 2016. [Online]. Available: http://dx.doi.org/10.1109/TAC.2015.2450571

[8] A. Levant, M. Livne, "Uncertain disturbances' attenuation by homogeneous MIMO sliding mode control and its discretization," IET Control Theory \& Applications, vol. 9, no. 4, pp. 515-525, 2015. [Online]. Available: http://dx.doi.org/10.1049/iet-cta.2014.0342

[9] A. Levant, "MIMO 2-sliding control design," Proc. Europian Control Conference ECC, Cambridge, UK, pp. 916-921, 1-4 Sept., 2003. [Online]. Available: http://dx.doi.org/10.23919/ECC.2003.7085075

[10] G. Bartolini, A. Ferrara, E. Usai, V. I. Utkin, "On multi input chattering free second order sliding mode control," IEEE Transactions on automatic Control, vol. 45, no. 9, pp. 1711-1717, Sept., 2000. [Online]. Available: http://dx.doi.org/10.1109/9.880629

[11] J. Ackermann, V. Utkin, "Sliding mode control design based on Ackermann's formula," IEEE Trans. on Automatic Control, vol. 43, no. 2, pp. 234-237, 1998. [Online]. Available: http://dx.doi.org/10.1109/9.661072

[12] B. Peruničić, Č. Milosavljević, B. Veselić, V. Gligić, "Comprehensive approach to sliding subspace design in linear time invariant systems," Proc. of IEEE 12th Int. Workshop on Variable Structure Systems (VSS 2012), pp. 473-478, Mumbai, India, 2012. [Online]. Available: http://dx.doi.org/10.1109/VSS.2012. 6163548

[13] B. Draženović, Č. Milosavljević, B. Veselić, "Comprehensive Approach to Sliding Mode Design and Analysis in Linear Systems”, in B. Bandyopadhyay, S. Janardhanan and S.K. Spurgeon (Eds.), Advances in Sliding Mode Control: Concept, Theory and Implementation, Lecture Notes in Control and Information Sciences, Vol. 440, Ch. 1, pp 1-19, Springer Berlin Heidelberg, 2013. [Online]. Available: http://dx.doi.org/10.1007/978-3-642-36986-5_1

[14] D. Hernandez, F. Castanos, L. Fridman, "Pole-placement in higher-order sliding-mode control," Proc. 19th IFAC Word Congres, pp. 1386-1391, 2014. [Online]. Available: http://dx.doi.org/10.3182/20140824-6-ZA1003.00949

[15] I. Castillo, F. Castaños, L Fridman, "Sliding Surface Design for Higher-Order Sliding Modes," in L. Fridman, J.P. Barbot, F. Plestan (eds.), Recent Trends in Sliding Mode Control, IET, 2016. [Online]. Available: https://doi.org/10.1049/PBCE102E_ch1.2 